\title{
RECOVERABILITY OF SOME CLASSES OF ANALYTIC FUNCTIONS FROM THEIR BOUNDARY VALUES*
}

\begin{abstract}
AHMED I. ZAYED
ABstract. The technique devised by D. J. Patil to recover the functions of the Hardy space $H^{p}(1 \leqslant p \leqslant \infty)$ from the restrictions of their boundary values to a set of positive measure on the unit circle was modified by S. E. Zarantonello in order to extend the result to $H^{p}(0<p<1)$.

In this paper, we show that Zarantonello's technique can be slightly modified to extend the result to a larger class of analytic functions in the unit disc. In particular, if $f(z)$ is analytic in the unit disc and satisfies
\end{abstract}

$$
\lim _{r \rightarrow 1}(1-r)^{\beta} \log M(r, f)=0 \text { for some } \beta \geqslant 1 \text {. }
$$

then $f(z)$ can be recovered from the restriction of its boundary value to an open arc.

1. Introduction. Let $\mathscr{D}$ denote the open unit disc, $\partial \mathscr{D}$ its boundary, i.e., the unit circle, and $\mu$ the normalized Lebesgue measure on $\partial \mathcal{Q}$. Furthermore, let $H^{p}(0<p \leqslant$ $\infty)$ denote the Hardy class of analytic functions in $\mathscr{Q}$. It is well known that if $f(z) \in H^{p}$ and $\lim _{r \rightarrow 1} f\left(r e^{i \theta}\right)=0$ on a set $E \subset \partial \mathscr{Q}$ of positive measure, then $f(z)$ is identically zero. We call this property the uniqueness property. In a sense, this means that functions in $H^{p}$ are uniquely determined by their values on $E$.

The question of whether a function $f \in H^{p}$ can be recovered from its restriction to $E$ was answered in the affirmative by D. Patil [1] for $1 \leqslant p \leqslant \infty$, who constructed a sequence of analytic functions that converged to $f$ uniformly on compact subsets of OD as well as in the norm. Modifying Patil's technique, S. Zarantonello [4] and G. Walker [3] independently were able to extend the results to $0<p<1$ but in a slightly more restrictive form.

A function $f(z) \in H^{p}(0<p<1)$ can be recovered from the restriction of its distributional boundary value to $E$ where $E$ now is an open arc in $\partial$ o 2 .

Since there are larger classes of analytic functions in of having the uniqueness property, e.g., the Nevanlinna class $N$, a natural question immediately arises, can functions in these classes be recovered from their restrictions to $E$ ? The purpose of this article is to show that the answer is "yes" for a large class of analytic functions. More precisely, if we denote by $\mathscr{H}(\alpha)(0<\alpha<1)$ the class of all analytic functions in $\mathcal{O}$ having the property $\int_{0}^{1} M(r, f) \exp \left(-c /(1-r)^{\beta}\right) d r<\infty$ for all $c>0$ where

This paper was presented at the 791st meeting of the American Mathematical Society in Santa Barbara, California, November 1981: received by the editors October 5, 1981.

1980 Mathematics Subject Classification. Primary 30E25: Secondary 30B30.

Key' n'ords and phrases. Recoverability theorem, Beurling distributions. Toeplitz operators.

*This paper previously appeared in Volume 86 of the Proceedings on pages 97-102. It is being reprinted because the pages appeared out of sequence. 
$M(r, f)=\sup _{|z|=r}|f(z)|$ and $\beta=\alpha /(1-\alpha)$, then any function $f(z) \in \mathcal{H}(1)=$ $\cup_{0<\alpha<1} \mathcal{H}(\alpha)$ can be recovered from the restriction of its distributional boundary value to an open arc $E$. The technique we use is a modification of the ones given by Patil and Zarantonello.

2. Preliminaries. For $0<\alpha<1$, let $\mathcal{H}(\alpha)$ be the space of all holomorphic functions $F(z)$ in $\mathscr{D}$ such that $F(z)=\sum_{n=0}^{\infty} a_{n} z^{n}$ with $a_{n}=O\left(e^{o\left(n^{\alpha}\right)}\right)$ as $n \rightarrow \infty$. The topology of $\mathcal{H}(\alpha)$ is the topology induced by the seminorms

$$
\|F\|_{c}=\sum_{n=0}^{\infty}\left|a_{n}\right| e^{-c n^{\alpha}}<\infty \quad \text { for } c>0 .
$$

In [5 and 6], we showed the following facts.

(1) $F(z) \in \mathscr{H}(\alpha)$ if and only if

$$
\left\|{ }_{\|}\right\|_{c}=\int_{0}^{1} M(r, f) \exp \frac{-c}{(1-r)^{\beta}} d r<\infty \quad \text { for all } c>0 .
$$

Moreover, the two families of seminorms $\|-\| \|_{c}$ and $\|-1\|_{c}$ are equivalent.

(2) Provided with that topology, $\mathcal{H}(\alpha)$ becomes a Fréchet-Montel space whose topology is stronger than the topology of uniform convergence on compact subsets of ol.

(3) If we denote by $Q_{\alpha}(0 D)$ the algebra of functions $G(z)$ that are analytic in on and continuous in $\bar{D}$ such that $G(z)=\sum_{n=0}^{\infty} b_{n} z^{n}, b_{n}=O\left(e^{-c n^{\alpha}}\right)$ as $n \rightarrow \infty$ for some $c>0$, then for any $\phi \in \mathcal{H}^{*}(\alpha)$ (the dual space of $\mathcal{H}^{-}(\alpha)$ ), there exists a unique function $G(z)=\sum_{n=0}^{\infty} b_{n} z^{n} \in \mathcal{G}_{\alpha}(\mathcal{O})$ ) such that

$$
\phi(F)=\lim _{r \rightarrow 1} \int_{\partial \cup \mathbb{Q}^{2}} F\left(r e^{i \theta}\right) G\left(e^{-i \theta}\right) d \mu=\sum_{n=0}^{\infty} a_{n} b_{n} .
$$

Conversely, any $G(z) \in \mathbb{Q}_{\alpha}(\cup \mathbb{L})$ defines a continuous linear functional on $\mathcal{H}(\alpha)$ via (2.2).

Let $G_{\alpha}^{\prime}=G_{\alpha}^{\prime}(\partial \cdot \mathcal{D})$ be the space of all $C^{\infty}$-functions $\phi$ on $\partial^{\mathcal{O} D}$ such that

$$
\phi(\theta)=\sum_{-\infty}^{\infty} b_{n} e^{i n \theta}
$$

with $b_{n}=O\left(e^{-\left\langle|n|^{\alpha}\right.}\right)$ as $n \rightarrow \infty$ for some $c>0$.

Another useful characterization of the class $Q_{\alpha}$ is that $\phi \in \mathbb{Q}_{\alpha}$ if and only if $\sup _{0 \leqslant \theta \leqslant 2 \pi}\left|\phi^{(n)}(\theta)\right| \leqslant K R^{n / \alpha} n^{n / \alpha}$ for some constants $K$ and $R$.

Indeed, the class $\mathbb{Q}_{\alpha}$ is the same as the class $C\left\{(n !)^{1 / \alpha}\right\}$ in the notation of [2].

It follows from the Denjoy-Carleman theorem that $\Theta_{\alpha}$ is a nonquasianalytic class of functions on $\partial \cup 2$. A topology is defined on $\vec{U}_{\alpha}^{\prime}$ by means of the fundamental system of neighborhoods of the origin

$$
V(\lambda)=\left\{\phi \mid \phi(\theta)=\sum_{-\infty}^{\infty} b_{n} e^{i n \theta}, b_{n}=O\left(e^{-\lambda_{n}|n|^{\alpha}}\right)\right\}
$$

where $\lambda=\left\{\lambda_{n}\right\}_{n=0}^{\infty}$ and $\lambda_{n} \downarrow 0$.

The strong dual $\mathbb{Q}_{\alpha}^{*}$ of $\mathcal{Q}_{\alpha}$ is a space of Beurling distributions which are more general than Schwartz distributions (see [5 and 6] for references). One can verify the following. 
(1) Every Beurling distribution $f$ has a Fourier series expansion $f=\sum_{-\infty}^{\infty} a_{n} w^{n}$ that converges weakly to it, where $w=e^{i \theta}$ and $a_{n}=\left\langle f, w^{n}\right\rangle$.

(2) A necessary and sufficient condition that $f=\sum_{-\infty}^{\infty} a_{n} w^{n} \in \mathbb{Q}_{\alpha}^{*}$ is that $a_{n}=$ $O\left(e^{o\left(|n|^{n}\right)}\right)$ as $n \rightarrow \infty$. Furthermore, if $\psi=\sum_{-\infty}^{\infty} b_{n} w^{n} \in\left(u_{\alpha}^{\prime}\right.$ then $\langle f, \psi\rangle=\sum_{-\infty}^{\infty} a_{n} \bar{b}_{n}$.

(3) If $F(z)=\sum_{n=0}^{\infty} a_{n} z^{n} \in \mathcal{H}(\alpha)$, then $f=\sum_{n=0}^{\infty} a_{n} w^{n}$ is a Beurling distribution belonging to the space $\mathcal{U}_{\alpha}^{*}$. In addition to that (cf. [5, Corollary 3.1])

$$
F_{r}(w)=F(r w) \rightarrow f \quad \text { in } c_{\alpha}^{*} .
$$

As in [4], we say that $f$ is the distributional boundary value of $F(z)$ and $F(z)$ is the holomorphic extension of $f$. The space of all distributional boundary values of functions in $\mathcal{H}^{*}(\alpha)$ will be denoted by $\tilde{\hat{\psi}}_{\alpha}^{*}$. We provide $\tilde{\psi}_{\alpha}^{*}$ with a topological structure isometric to that of $\mathcal{K}(\alpha)$ by setting

$$
\|f\|_{c}=\|F\|_{\text {. }} .
$$

From (2.1) and (2.2), one can easily see that the topology of $\bar{c}_{\alpha}^{*}$ is stronger than the topology it inherits from ( $_{\alpha}^{*}$.

From Cauchy's formula

$$
F(r z)=\int_{\partial w^{\prime}} \frac{F_{r}\left(w^{\prime}\right)}{1-\overline{w^{\prime} z}} d \mu\left(w^{\prime}\right)
$$

and equation (2.3), it follows that $F(z)=\left\langle f, C_{z}\right\rangle$ where $C_{z}(w)=1 /(1-w \bar{z})$.

3. Toeplitz operators on $\tilde{\psi}_{\alpha}^{*}$. Since $\mathcal{Q}_{\alpha}$ is an algebra [2], we define the multiplication operator $M_{\phi}$ for $\phi \in \mathbb{Q}_{\alpha}$ by

$$
\left\langle M_{\phi} f, \psi\right\rangle=\langle f, \bar{\phi} \psi\rangle
$$

where $f \in \mathbb{U}_{\alpha}^{*}$ and $\psi \in \mathbb{U}_{\alpha}$.

The projection operator $P: \mathbb{\mathcal { A }}_{\alpha}^{*} \rightarrow \tilde{\mathbb{Q}}_{\alpha}^{*}$ is formally given by

$$
P\left(\sum_{-\infty}^{\infty} a_{n} w^{n}\right)=\sum_{0}^{\infty} a_{n} w^{n}
$$

and hence the Toeplitz operator $S_{\phi}$ can now be defined on $\tilde{\mathbb{C}}_{\alpha}^{*}$ as $S_{\phi}=P M_{\phi}$ for $\phi \in \hat{U}_{\alpha}$.

LEMmA 1. Let $\phi \in \mathbb{Q}_{\alpha}$ and $f \in \tilde{\mathbb{U}}_{\alpha}^{*}$, then:

(i) For any $c>0$ there exists $\tilde{c}$ such that

$$
\left\|S_{\phi} f\right\|_{c} \leqslant K(\phi, c)\|f\|_{\tilde{c}}
$$

where $K(\phi, c)$ is a constant that depends only on $c$ and $\phi$ but. not on $f$.

(ii) $S_{\phi} f$ is the distributional boundary value of the analytic function $\left\langle M_{\phi} f, C_{z}\right\rangle$.

Proof. (i) Let $f=\sum_{0}^{\infty} a_{n} w^{n}$ and $\phi=\sum_{-\infty}^{\infty} b_{n} w^{n}$, then $S_{\phi} f=\sum_{k=0}^{\infty} \sum_{n=0}^{\infty} a_{n} b_{k-n} w^{k}$. Hence,

$$
\begin{aligned}
\left\|S_{\phi} f\right\|_{c} & \leqslant \sum_{k=0}^{\infty} \sum_{n=0}^{\infty}\left|a_{n}\right|\left|b_{k-n}\right| e^{-c|k|^{\alpha}}=\sum_{n=0}^{\infty}\left|a_{n}\right| \sum_{k=-n}^{\infty}\left|b_{k}\right| e^{-c|k+n|^{\alpha}} \\
& =\sum_{n=0}^{\infty}\left|a_{n}\right| \sum_{k=-n}^{-1}\left|b_{k}\right| e^{-c|k+n|^{\alpha}}+\sum_{n=0}^{\infty}\left|a_{n}\right| \sum_{k=0}^{\infty}\left|b_{k}\right| e^{-c|k+n|^{\alpha}}=I_{1}+I_{2} .
\end{aligned}
$$


But $(k+n)^{\alpha} \leqslant k^{\alpha}+n^{\alpha} \leqslant 2(k+n)^{\alpha}$, therefore

$$
I_{2} \leqslant \sum_{n=0}^{\infty}\left|a_{n}\right| \sum_{k=0}^{\infty}\left|b_{k}\right| \exp \left(-\frac{1}{2} c|k|^{\alpha}-\frac{1}{2} c|n|^{\alpha}\right) \leqslant B\|f\|_{c / 2}
$$

where $B=\sum_{k=0}^{\infty}\left|b_{k}\right| \exp \left(-\frac{1}{2} c|k|^{\alpha}\right)$ which certainly converges since $\left|b_{k}\right| \leqslant A e^{-h|k|^{\alpha}}$ for some $b>0$. On the other hand,

$$
\begin{aligned}
I_{1} & \leqslant \sum_{n=0}^{\infty}\left|a_{n}\right| \sum_{k=-n}^{-1} A e^{n|k|^{n}-c|k+n|^{n}} \\
& \leqslant A \sum_{n=0}^{\infty}\left|a_{n}\right| n e^{-2 a|n|^{n}} \leqslant \tilde{B} \sum_{n=0}^{\infty}\left|a_{n}\right| e^{-d|n|^{n}}=\tilde{B}\|f\|_{d}
\end{aligned}
$$

where $d=\frac{1}{2} \min (b, c)$ and $\tilde{B}=\max _{0 \leqslant n} A\left(n / e^{d n^{a}}\right)<\infty$.

From (3.1), (3.2) and (3.3), it follows that $\left\|S_{\phi} f\right\|_{c} \leqslant K\|f\|_{\tilde{\tau}}$ where $K=\max (B, \tilde{B})$ and $\tilde{c}=\min (c / 2, d)$.

(ii) Let $f \in \mathcal{H}(\alpha)$ and $F(z)$ be its holomorphic extension to $0 \mathrm{D}$. From Corollaries 3.1 and 3.2 of [5], it follows that $F_{r}(w)=F(r w) \rightarrow f$ in $\Re(\alpha)$ and that $H^{2}$ is dense in $\Re(\alpha)$. Moreover, $F_{r}(w)$ is the boundary value of a function in $H^{2}$, namely $F_{r}(z)$. Now an argument similar to that of Theorem 3.2 of [4] finishes the proof.

4. Recoverability Theorem. In this section we shall use the same notation as [4]. Let $E$ be an open arc in $\partial \cup \mathcal{D}$ and $\psi \in \mathcal{U}_{\alpha}$ such that $0 \leqslant \psi \leqslant 1$, supp $\psi \subset E$ and

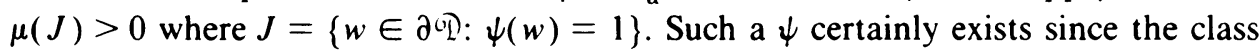
$\dot{u}_{\alpha}^{\prime}$ is nonquasianalytic. For each $0<\lambda<1$ define

$$
\phi_{\lambda}(w)=\frac{1}{1-\lambda \psi(w)}
$$

and

$$
H_{\lambda}(z)=\exp \left\{-\frac{1}{2} \int_{\partial \cdot \mathrm{Q}} \frac{w+z}{w-z} \log \phi_{\lambda}(w) d \mu\right\}, \quad z \in \mathrm{NQ} .
$$

As is shown in [1 and 4], if we denote the boundary value of $H_{\lambda}(z)$ by $h_{\lambda}(w)$, we have

(a) $\left|h_{\lambda}(w)\right|^{-2}=\phi_{\lambda}(w)$.

(b) $h_{\lambda}$ and $h_{\lambda}^{-1}$ are in $H^{\infty}(\partial \cdot 1)$ ).

LEMMA 2. (i) $\phi_{\lambda} \in \mathbb{U}_{\alpha^{*}}$.

(ii) $S_{\phi_{\lambda}}: \tilde{\mathbb{U}}_{\alpha}^{*} \rightarrow \tilde{\mathbb{Q}}_{\alpha}^{*}$ is invertible with inverse $S_{\phi_{\lambda}}^{-1}=S_{h_{\lambda}} S_{h_{\lambda}}^{-}$.

(iii) For any $c>0$, there exists $\tilde{c}$ such that

$$
\left\|S_{\phi_{\lambda}}^{-1} f\right\|_{c} \leqslant K\|f\|_{\tilde{c}} \text { where } K \text { is independent of } \lambda \text {. }
$$

Proof. (i) Since $\psi \in \mathbb{Q}_{\alpha}$ and $\mathbb{Q}_{\alpha}$ is an inverse-closed, nonquasianalytic class of functions [2], it follows immediately that $\phi_{\lambda} \in \mathcal{Q}_{\alpha}$.

(ii) It suffices to show that $h_{\lambda} \in \mathbb{Q}_{\alpha}$. Let $h_{\lambda}(w)=e^{-\left(u\left(w^{\prime}\right)+i v\left(w^{\prime}\right)\right)}$. Since $\log \phi_{\lambda}(w) \in$ $Q_{\alpha}$ by part (i) and Theorem A of [2], it is easy to see that $u(w)$ and $v(w)$ are also in $\mathbb{Q}_{\alpha}$. We invoke Theorem A once more to show that $h_{\lambda}$ is indeed in $\mathbb{Q}_{\alpha}$.

(iii) $\left\|S_{h_{\lambda}} S_{h_{\lambda}}^{-} f\right\|_{c} \leqslant \sum_{k=0}^{\infty} \sum_{m=0}^{\infty} \sum_{n=0}^{\infty}\left|\hat{h}_{\lambda}(k) \| \overline{\hat{h}_{\lambda}}(m)\right||\hat{f}(n)| e^{-c|k-m+n|^{\alpha}}$. 
Since $\left|h_{\lambda}(w)\right| \leqslant\left(\phi_{\lambda}(w)\right)^{-1 / 2} \leqslant 1$, it follows that the sequence $\left\{\hat{h_{\lambda}}(k)\right\}$ is uniformly bounded. Now an argument similar to the one given in Lemma 1 yields the result.

LEMMA 3. For fixed $z \in \mathscr{D}$ and $c>0, \lim _{\lambda \rightarrow 1}\left\|S_{\phi_{\lambda}}^{-1} C_{z}\right\|_{c}=0$.

Proof. The relation (cf. [4, equation (4.4.2)])

$$
\left|H_{\lambda}(z)\right|=\exp \left\{-\frac{1}{2} \int \frac{1-|z|^{2}}{|1-\bar{w} z|^{2}} \log \phi_{\lambda}(w) d \mu(w)\right\} \leqslant(1-\lambda)^{\alpha}
$$

where $2 \alpha=\{(1-|z|) /(1+|z|)\} \mu(J)>0$, shows that $H_{\lambda}(z) \rightarrow 0$ uniformly on compact subsets of $\mathscr{D}$ as $\lambda \rightarrow 1$. Since $H_{\lambda}(z)=\sum_{k=0}^{\infty} \hat{h}_{\lambda}(k) z^{k}$, it is routine to show that $\hat{h}_{\lambda}(k) \rightarrow 0$ as $\lambda \rightarrow 1$ for $k=0,1,2, \ldots$ Moreover, $\left|\hat{h}_{\lambda}(k)\right| \leqslant B$ for all $0<\lambda<1$ and $k=0,1,2, \ldots$

We claim that $H_{\lambda}(z) \rightarrow 0$ in $\mathcal{H}(\alpha)$ as $\lambda \rightarrow 1$. For

$$
\left\|H_{\lambda}\right\|_{c}=\sum_{k=0}^{\infty}\left|\hat{h}_{\lambda}(k)\right| e^{-c k^{\alpha}}=\sum_{k=0}^{N}\left|\hat{h}_{\lambda}(k)\right| e^{-c k^{\alpha}}+\sum_{N+1}^{\infty}\left|\hat{h}_{\lambda}(k)\right| e^{-c k^{\alpha}} \text {. }
$$

Choose $N$ large enough so that $\sum_{N+1}^{\infty} e^{-c k^{\alpha}} \leqslant \varepsilon / 2 B$ and $\lambda$ so close to 1 that $\left|\hat{h_{\lambda}}(k)\right| \leqslant \varepsilon / 2(N+1)$ for $k=0,1, \ldots, N$. Substitution in (4.1) gives

$$
\left\|H_{\lambda}\right\|_{c} \leqslant \varepsilon \text { for } \lambda \text { sufficiently close to } 1 \text {. }
$$

By an argument similar to the one given in Lemma 1 , the fact that $S_{\phi_{\lambda}}^{-1} C_{z}=$ $\bar{H}_{\lambda}(z) h_{\lambda} C_{z}$ and (4.2) one easily obtains

$$
\lim _{\lambda \rightarrow 1}\left\|S_{\phi_{\lambda}}^{-1} C_{z}\right\|_{c}=\lim _{\lambda \rightarrow 1}\left\|\bar{H}_{\lambda}(z) h_{\lambda} C_{z}\right\|_{c} \leqslant A \lim _{\lambda \rightarrow 1}\left\|H_{\lambda}\right\|_{\tau}=0
$$

for some $\tilde{c}>0$ and a constant $A$ that depends on $z$ and $c$ but not on $\lambda$.

LEMMA 4. Let $f \in \tilde{\mathbb{Q}}_{\alpha}^{*}$ and $g_{\lambda}=S_{\phi_{\lambda}}^{-1}\left(S_{\phi_{\lambda}}-I\right) f$, then $\lim _{\lambda \rightarrow 1} g_{\lambda}=f$ in $\tilde{\mathbb{U}}_{\alpha}^{*}$.

Proof. As a consequence of Corollary 3.2 of [5], $L^{\infty}(\partial \mathcal{D})$ is dense in $\tilde{\mathbb{P}}_{\alpha}^{*}$. From this and the fact that $\left\{C_{z}: z \in \mathscr{D}\right\}$ is a fundamental set in $L^{2}(\partial \mathcal{D})$ and that the embedding $L^{2}(\partial \cup \mathcal{}) \rightarrow \tilde{Q}_{\alpha}^{*}$ is continuous, we conclude that $\left\{C_{z}: z \in \cup \mathbb{R}\right\}$ is also a fundamental set in $\tilde{\mathcal{G}}_{\alpha}^{*}$. This fact together with Lemmas 2 and 3 may now be used to show that $\lim _{\lambda \rightarrow 1}\left\|S_{\phi_{\lambda}}^{-1} f\right\|_{c}=0$. But $S_{\phi_{\lambda}}^{-1} f=f-g_{\lambda}$ which yields the result.

Using arguments parallel to those given in [ 4 and 1 ], the reader should be able to finish the proof of the following theorem.

THEOREM. Let $E$ be an open arc of $\partial \cup \mathrm{L}$. Let $F(z) \in \mathcal{H}(\alpha)$ and $f$ be its distributional boundary value on $\partial \cup \mathrm{L}$. Suppose that $g$ is the restriction of $f$ to $E$. For each $0<\lambda<1$ define an analytic function $G_{\lambda}(z)$ on ol $_{2}$ by

$$
G_{\lambda}(z)=H_{\lambda}(z)\left\langle g,\left(\phi_{\lambda}-1\right) h_{\lambda} C_{z}\right\rangle \text {. }
$$

Then, $\lim _{\lambda \rightarrow 1} G_{\lambda}(z)=F(z)$ in $\mathcal{K}(\alpha)$. In particular, $\lim _{\lambda \rightarrow 1} G(z)=F(z)$ uniformly on compact subsets of $\mathrm{Dl}$.

It is well known that functions in $\mathcal{H}(\alpha)$ can have radial limits equal to zero on a set $E$ of positive measure (in fact, even on a set of measure $2 \pi$ ) without being 
identically zero i.e., the class $\mathcal{H}(\alpha)$ does not have the uniqueness property. However, the class $\mathcal{H}(\alpha)$ possesses another uniqueness property which we state as a corollary.

COROllary. Let $F(z) \in \mathcal{H}(\alpha)$ and $f$ be its distributional boundary value. If $f=0$ (in the sense of distributions) on an open arc $E$, then $F(z)$ is identically zero.

RemarKs. (i) One may ask how far can the result be extended? To answer this question, let us consider the class $\mathcal{H}(\omega)$ of all analytic functions $F(z)=\sum_{n=0}^{\infty} a_{n} z^{n}$ in $\mathscr{D}$ with $a_{n}=O\left(e^{o(\omega(n))}\right)$ as $n \rightarrow \infty$ where $\omega(x)$ is continuously differentiable and monotonically increasing on $[0, \infty)$. Certainly, our technique fails if $\sum_{n=0}^{\infty} \omega(n) / n^{2}$ $=\infty$, since in this case the class $\mathcal{Q}_{\omega}$ is quasianalytic by the Denjoy-Carleman theorem. Therefore, if the function $\psi(w)=0$ on a set of positive measure, it is identically zero on $\partial \mathscr{D}$ and hence $G_{\lambda}(z)$ is identically zero for all $0<\lambda<1$.

(ii) The class $N^{+}$provided with the topology induced by the metric

$$
(f, g)=\int_{\partial \mathcal{D}} \log \left(1+\left|f\left(e^{i \theta}\right)-g\left(e^{i \theta}\right)\right|\right) d \mu
$$

where $f$ and $g \in N^{+}$is a subspace of $\mathcal{H}(1 / 2)$.

From the main theorem, it follows that if $F \in N^{+}$, then $G_{\lambda}(z) \rightarrow F(z)$ in $\mathcal{H}(1 / 2)$. It would be interesting to know whether $G_{\lambda}(z) \rightarrow F(z)$ in $N^{+}$.

ACKNOWLedgement. The author wishes to thank Professor Walter Rudin for bringing to his attention the results of reference [2].

\section{REFERENCES}

1. D. Patil, Representation of $H^{p}$-functions, Bull. Amer. Math. Soc. 4 (1972), 617-620.

2. W. Rudin, Division in algebras of infinitely differentiable functions, J. Math. Mech. 11 (1962), 797-810.

3. G. Walker, Analvtic representations, values, and recoverability of distributions, $\mathrm{Ph}$. D. thesis, University of Wisconsin, Milwaukee, 1974.

4. S. Zarantonello, $A$ representation of $H^{p}$ functions with $0<p<\infty$, Pacific J. Math. 79 (1978), $271-282$.

5. A. Zayed, Topological vector spaces of analytic functions, submitted.

6. On Beurling distributions and holomorphic functions in the unit disc, submitted.

Department of Mathematics, California Polytechnic State University, San luis Obispo, CALIFORNIA 93407 